\title{
The Filtration Characteristics of the Aqueous Outflow System
}

\author{
MARK JOHNSON ${ }^{a, b}$, DOUGLAS H. JOHNSON ${ }^{c}$, ROGER D. KAMM ${ }^{a}$, \\ ANNELIES W. DEKATER ${ }^{b}$ AND DAVID L. EPSTEIN ${ }^{b}$ \\ ${ }^{a}$ Massachusetts Institute of Technology. Cambridge. MA, ' Howe Laboratory of Ophthalmology, \\ Massachusetts Eye and Ear Infirmary, Harvard Medical School, Boston, MA, and 'Mayo Clinic, \\ Rochester, MN, U.S.A.
}

(Received 21 June 1989 and accepted in revised form 15 September 1989)

\begin{abstract}
To determine the filtration characteristics of the aqueous outflow system, microspheres $(0 \cdot 18 \mu \mathrm{m}-1 \cdot 1 \mu \mathrm{m})$ werc perfused through enucleated human and bovine cycs. The microspheres were smaller than morphologically determined flow dimensions, and yet a significant fraction of all sizes of microspheres were captured. The bovine (calf) aqueous outflow system was found to be a far more efficient filter than was the human outflow system.

Combining the experimental results with morphological observations and theoretical calculations leads to the conclusion that 'sticky wall' interactions are responsible for much of the microsphere capture, and that the site of filtration may be distinct from the site of flow resistance. Consequently. the dimension of the sites generating flow resistance cannot be determined from filtration studies.

Key words: filtration; trabecular meshwork; microspheres; human; bovine.
\end{abstract}

\section{Introduction}

Many investigators have studied the filtration ${ }^{1}$ characteristics of the aqueous outflow system with the goal of determining the caliber of the openings that generate aqueous outtlow resistance. In the 1870 s, Schwalbe and Leber disagreed as to whether colloidal suspensions could pass through this system (Huggert, 1955). Through the next $50 \mathrm{yr}$, the conclusion was reached that small particles may successfully pass through the outflow system, while larger particles are captured, thus verifying Schwalbe's contention that there are pores in the filtration apparatus.

Huggert conducted a number of studies (Huggert. 1955. 1957; Huggert, Holmberg, and Eklund, 1955) aimed at determining the pore size distribution in the aqueous outflow system. Huggert hoped to characterize the pore size distribution within the filtration apparatus by perfusing the eye with particulate solutions. Comparing the distribution of particlcs sizes leaving the aqueous outflow system (collected as they emerged from the aqueous veins) to that entering the anterior chamber, he hoped to deduce the pore size distribution.

Huggert assumed that the particles were captured by the filtration apparatus through steric hindrance: the pores were too small for the particles to pass through. He used his results to conclude that a heteroporosity of pore sizes existed with a maximum pore size of several microns but with most pores an order of magnitude smaller.

Barany (1959) reanalyzed Huggert's data assuming that the capture was not due to steric hindrance as Huggert assumed. but instead due to the particles

\footnotetext{
${ }^{1}$ By filtration. we mean the collection of particles contained in the perfusion fluid.
}

adhering to the boundaries of the outflow pathway, reasoning that the endothelium of the trabecular meshwork must be 'sticky' for the purpose of phagocytosis. He found that using this assumption, the data were consistent with the existence of pores approximately $1 \mu \mathrm{m}$ in diameter.

These differing assumptions regarding capture modes are quite important in the evaluation of experimental results. Huggert's model of capture by steric hindrance predicts that below a certain critical size, all particles should successfully pass the system; Barany's model predicts significant capture for all sizes: large particles captured by direct interception onto the sticky walls while small particles have such large Brownian motion that they are also likely to hit the sticky walls. These models thus give very different predictions for the fraction of particles filtered for very small particle sizes. We undertook the current study to determine which of these models better describes the filtration characteristics of the aqueous outflow system.

An additional discrepancy we investigated involves the work of Karg et al. (1959). They performed a study similar to that of Huggert using latex microspheres in human eyes. In contrast to Huggert's results, they found that particles smaller than $1.5 \mu \mathrm{m}$ easily passed through the outflow system. However. Inomata, Bill and Smelser (1972) perfused primate eyes with a microsphere solution containing $0 \cdot 1-, \quad 0.5-$ and $1 \cdot 0-\mu \mathrm{m}$ microspheres and examined these eyes histologically. They found little capture of $0 \cdot 1-\mu \mathrm{m}$ microspheres, but found many 0.5 - and $1 \cdot 0-\mu \mathrm{m}$ microspheres piled up at the entrance to the juxtacanalicular connective tissue (JCT), thus indicating significant filtration. It was a further goal of the present study to resolve this discrepancy. 


\section{Materials and Methods}

We perfused solutions containing microspheres through the outflow system of enucleated bovine (calf) and human eyes, and collected the perfusate as it emerged onto the sclera. The change in concentration of microspheres in the perfusion fluid as it passed through the aqueous outflow system was then determined.

Calf eyes were typically used within 3-4 hr of death. In two experiments, 1- and 2-day-old calf eyes were deliberately used to ascertain the effects of postmortem changes. Normal human autopsy eyes were obtained, within 12-36 hr of death, from the New England Eye Bank or through NDRI (National Disease Research Interchange, Philadelphia, $\mathrm{P} \Lambda$ ).

\section{Perfusion Fluid}

The perfusion fluid, Dulbecco's phosphatc buffered saline (Life Science Technologies Inc., Chagrin Falls, $\mathrm{OH}$ ), was prepared with fluorescein (Coopervision. San German, Puerto Rico) (one drop of $2 \%$ fluorescein/ $20 \mathrm{ml}$ buffer) and glucose $(5.5 \mathrm{~mm})$ and filtered through a $0.08-\mu \mathrm{m}$ Nucleopore filter (Nucleopore Corp., Pleasanton, CA). Fluorescein was added to allow easy visualization and collection of the samples. One $\mu \mathrm{l}$ of a $10 \%$ solution of $0.176-\mu \mathrm{m}$ microspheres and/or $3 \mu \mathrm{l}$ of a $10 \%$ solution of $0.46-\mu \mathrm{m}$ microspheres were added to $100 \mathrm{ml}$ of the perfusion fluid to obtain a volume fraction of microspheres in the perfusion fluid of approximately $4 \times 10^{-6}$. This very low concentration of microspheres was chosen to be sufficiently dilute to preclude the possibility that captured microspheres would occlude the outflow pathway and change its filtration characteristics.

\section{Experimental Procedure}

Eyes were placed in a funnel, cannulated and then submerged in silicone oil (Dow Corning 710 fluid). The cye was secured with a suture through the optic nerve. The perfusion pressure was adjusted to be between 6 and $23 \mathrm{mmHg}$ (see Table I). In early experiments, the perfusion needle was placed into the posterior chamber of the eye to avoid artificially deepening the anterior chamber and thus decreasing outflow resistance (Moses, 1977); however, since no significant difference was seen in the percentage of microspheres passing through the aqueous outflow system with the needle in the anterior chamber versus the posterior chamber (Johnson, 1987), most of the later experiments had the infusion needle in the anterior chamber. Details can be found in Table I.

The heavier-than-water immiscible silicone oil caused the perfusate to form bubbles as it exited the collector channels at the sclera, allowing the perfusate to be collected from the surface of the eye using gentle suction through a Pasteur pipette. Since the viscosity of the oil is much higher than that of the perfusate. very little oil entered the Pasteur pipette. The fluid collected during this first period is rejected to avoid errors associated with dilution effects. Thereafter. samples were collected approximately every 30 (calf) or 60 (human) min and saved for processing.

Samples of perfusion fluid entering the aqueous outflow system were collected using one of several procedures. In the earliest experiments, only a measurement of the concentration of microspheres entering the anterior chamber was taken. This control group is identified as Baseline in Table I. In later experiments, two different procedures were used. In the first, a sample of fluid was taken from the anterior chamber at the conclusion of the experiment. In the second, a second needle was placed into the anterior chamber at the beginning of the experiment. The needle was used to exchange the contents of the anterior chamber and could also be used to periodically sample the concentration of microspheres in the anterior chamber. These latter methods are identified as AC or Exchange in Table I. Both methods gave comparable results and thus the results of the latter two procedures were poolcd.

\section{Determination of Microsphere Concentration}

All samples were briefly centrifuged ( $5-10 \mathrm{sec}$ ) at $15000 \mathrm{~g}$ to separate any oil that might have been collected with the sample. (Samples collected from the anterior chamber were first mixed with a little oil to duplicate the conditions under which the perfusate was collected.)

A Nuclcopore polycarbonate filter $(0.08$ or $0.2 \mu \mathrm{m}$, depending on the particle size being used) was placed in a holder (Millipore, part no. SX0001300) that was then filled with distilled, deionized water that had been prefiltered through a $0 \cdot 08-\mu \mathrm{m}$ filter. Forty $\mu \mathrm{l}$ of the sample were added, and then $2 \mathrm{ml}$ of filtered water was pushed through the filter, followed by $1 \mathrm{ml}$ of air: this procedure spreads the microspheres over the surface of the filter while washing away the saline buffer. The filter was then prepared for scanning electron microscopy (SEM).

To determine the density of microspheres covering the surface of the filter, the button was placed in a JEOL JSM-35 scanning electron microscope, and the density of microspheres were determined in approximately 20 randomly selected regions of the filter. Magnifications of $1000 \times, 2000 \times$ and $4400 \times$ were used for counting the $1 \cdot 1-\mu \mathrm{m}, 0 \cdot 46-\mu \mathrm{m}$ and $0 \cdot 176-\mu \mathrm{m}$ microspheres, respectively. Typically, baseline samples had 50-200 microspheres per region counted.

\section{Statistical Methods}

Statistical treatment of the data generated several levels of variability - variability in the determination 
TABLE I

Parameters for microsphere perfusions

\begin{tabular}{|c|c|c|c|c|c|c|}
\hline Experiment $^{\alpha}$ & Eyes & Microspheres $(\mu \mathrm{m})^{l}$ & $\begin{array}{c}\text { Control } \\
\text { solution }^{b}\end{array}$ & Needle $^{c}$ & $\Delta \mathrm{P}(\mathrm{mmHg})$ & Notes \\
\hline $1 a, b$ & Calf & $0 \cdot 46$ & Baseline & $\mathrm{PC}$ & 6 & $" d$ \\
\hline $2 a, b$ & Human & 0.46 & Baseline & $\mathrm{PC}$ & N.D. & - \\
\hline $3 a, b$ & Calf & $0 \cdot 176$ & Baseline & $\mathrm{PC}$ & 7 & $\ldots$ \\
\hline $4 a$ & Calf & 0.176 & Baseline & $\mathrm{PC}$ & 13 & \\
\hline $4 b$ & Calf & $0 \cdot 176$ & Baseline & $\mathrm{PC}$ & 8 & - \\
\hline $5 a$ & Human & $0 \cdot 46$ & Baseline & $P C$ & 12 & - \\
\hline $6 a$ & Calf & $0 \cdot 176,0 \cdot 46$ & $A C$ & $\mathrm{PC}$ & $7 \cdot 5$ & - \\
\hline $6 b$ & Calf & $0 \cdot 176$ & $\mathrm{AC}$ & $\mathrm{PC}$ & $7 \cdot 5$ & $-\ldots$ \\
\hline $7 a$ & Human & $0.176,0.46$ & Baseline & $\mathrm{PC}$ & 6.5 & - \\
\hline $7 \mathrm{~b}$ & Human & $0 \cdot 176$ & $\mathrm{AC}$ & $\mathrm{PC}$ & $6 \cdot 5$ & - \\
\hline $8 \mathrm{a}$ & Human & $0 \cdot 176.0 \cdot 46$ & $\mathrm{AC}$ & $\mathrm{PC}$ & $10-15$ & " \\
\hline $10 a$ & Calf & $0 \cdot 176$ & $\mathrm{AC}$ & $\mathrm{PC}$ & 17 & - \\
\hline $10 \mathrm{~b}$ & Calf & $0 \cdot 176$ & $A C$ & $\mathrm{AC}$ & 21 & - \\
\hline $12 \mathrm{a}$ & Calf & $0 \cdot 176,0 \cdot 46$ & Exchange & $A C$ & 18 & - \\
\hline $12 \mathrm{~b}$ & Calf & $0 \cdot 176,0 \cdot 46$ & Exchange & $\mathrm{PC}$ & 18 & - \\
\hline $13 a$ & Calf & $0 \cdot 176,0 \cdot 46$ & Exchange & $\mathrm{AC}$ & $16 \cdot 5$ & - \\
\hline $13 \mathrm{~b}$ & Calf & $0 \cdot 176.0 \cdot 46$ & Exchange & $\mathrm{PC}$ & $16 \cdot 5$ & - \\
\hline $14 a$ & Calf & $0 \cdot 176,0 \cdot 46$ & AC & $\mathrm{PC}$ & $11-23$ & t \\
\hline $15 a$ & Calf & $0 \cdot 176,0.46$ & $\mathrm{AC}$ & $\mathrm{PC}$ & 21 & - \\
\hline $15 b$ & Calf & $0 \cdot 176,0.46$ & Exchange & $\mathrm{PC}$ & 18 & -- \\
\hline $16 a$ & Human & $0.176,0.46$ & Exchange & $\mathrm{AC}$ & N.D. & - \\
\hline $17 \mathrm{a}$ & Calf & $0 \cdot 46$ & Exchange & $A C$ & 11 & y \\
\hline $26 a, b$ & Calf & $0 \cdot 46$ & $\mathrm{AC}$ & $A C$ & 17 & $"$ \\
\hline $26 \mathrm{c}, \mathrm{d}$ & Calf & 0.5 & $A C$ & $\mathrm{AC}$ & 17 & $i$ \\
\hline $29 a, b$ & Calf & $0 \cdot 3,1 \cdot 1$ & Exchange & $\mathrm{AC}$ & 10 & j \\
\hline $30 a, b$ & Calf & $0 \cdot 176,0 \cdot 46$ & Exchange & $\mathrm{AC}$ & $9 \cdot 5$ & " \\
\hline $32 a$ & Human & $0 \cdot 176,0 \cdot 46$ & $\mathrm{AC}$ & $\mathrm{AC}$ & 11 & - \\
\hline 34a & Human & $0 \cdot 176,0 \cdot 46$ & Exchange & $\mathrm{PC}$ & 12 & - \\
\hline $34 b$ & Human & $0.176,0.46$ & Exchange & $A C$ & 10 & - \\
\hline
\end{tabular}

${ }^{a}$ Experiment numbers are listed consistent with Johnson (1987). a,b refer to the two eyes of a pair.

${ }^{b}$ Baseline is from reservoir entering the anterior chamber: $\mathrm{AC}$ is from the anterior chamber. Exchange results from an exchange of the anterior chamber.

cPerfusion needle position: $\mathrm{AC}$ or $\mathrm{PC} . \quad{ }^{d}$ 2-day-old eyes. ${ }^{e}$ Glaucomatous eye. ${ }^{f} \Delta \mathrm{P}$ initially 11 , then raised to 23.

g Carboxylate-labeled microspheres.

${ }^{h}$ Collection technique was altered in this experiment. Each eye was placed in plastic bags, perfused and the perfusate was then collected (Johnson and Kamm, 1987).

i Carboxylate-labeled microspheres. Collection procedure as in note $h,{ }^{j} 0 \cdot 3 \mu \mathrm{m}$ microspheres were carboxylate-labeled. ${ }^{k}$ l-day-old eyes.

' Microspheres used were polystyrene latex microspheres originally manufactured by Dow Chemical but now distributed by Duke Scientific (Palo Alto, CA): $0 \cdot 176-\mu \mathrm{m}$ microspheres (part no. 5018A) and 0.46- $\mu \mathrm{m}$ microspheres (part no. 5046A): 1.1- $\mu \mathrm{m}$ microspheres were manufactured by Duke (part no. 5100A). Carboxylate modified latex microspheres included 0.46- $\mu \mathrm{m}$ microspheres (part no. C046A) from Duke Scientific, and fluorescently-labeled 0.3- $\mathrm{m}$ (part no. 102025A), 0.5- $\mu \mathrm{m}$ (part 102050A) and $1 \cdot 0-\mu \mathrm{m}$ (part no. 102030A) microspheres from Covasphere Technologies (Ann Arbor, MI).

of microsphere concentration for a particular sample, variability in concentration of microspheres passing through an individual eye, and variability inherent within a population of eyes perfused with a particular size of microsphere.

The distribution of microsphere density found on the Nucleopore filter surface is expected to be Poisson. describing a random arrival process. For a particular sample, the mean and standard error of the number of microspheres found in each of the 20 selected regions on the filter were determined. A coefficient of variation (standard deviation/mean) was also determined in a few experiments by making multiple determinations of microsphere concentration for a particular solution.

To characterize the variability in concentration of microspheres passing through the outflow system of a particular eye, a number of successive measurements
( $m$ ) was taken, and for those cases that $m>1$, a mean and standard error were determined. To detcrmine the variability within a population of eyes (for a given size microsphcre), a mcan and standard deviation were computed. The standard deviation, rather than the standard error was used as the variabilities that resulted from this procedure were larger than would be cxpected based on the standard errors of the individual eyes (as determined using an analysis of variance). These variabilities are likely due to variations within the population, and thus the standard deviation is the proper statistical measure.

\section{Morphology}

To determine where in the aqueous outflow system the microspheres were being caught, several eyes were 
examined morphologically. A modification of the method of Inomata et al. (1972) was followed. Briefly, the system was perfused with microspheres for several hours (using the low concentration of microspheres described above) followed by an exchange of the anterior chamber with a gelatin solution $(2.5 \%$ gelatin in Dulbecco's phosphate buffered saline with $5.5 \mathrm{mM}$ glucose kept at $35^{\circ} \mathrm{C}$ until infusion). A $2.5 \%$ solution was chosen, as we were unable to demonstrate that a $5 \%$ solution (as used by Inomata et al., 1972) would enter and flow through the aqueous outflow system of a bovine eye. The perfusion with gelatin solution was continued for 30-60 min. This served to both fill the extracellular spaces with gelatin (allowing the microsphere locations to be identified, as these are dissolved during specimen preparation) and also to wash-out any microspheres that were in transit.

The eye was then chilled with ice for 15 min and placed in cold fixative ( $2 \%$ paraformaldehyde, $2.5 \%$ glutaraldehyde in Sorenson's phosphate buffer). bisected at the equator, and angle tissue was dissected from each of the four quadrants. Specimens were immersed in fixative for an additional $4 \mathrm{hr}$, washed in Sorenson's buffer, postfixed in $1 \%$ osmium tetraoxide in Sorenson's buffer, dehydrated in graded alcohols and embedded in Epon-Araldite. Ultrathin sections were cut on a LKB ultramicrotome, stained with uranyl acetate and lead acetate, and finally examined on a JEOL JEM 7 or Philips CM10 transmission electron microscope.

\section{Results}

Experiments were conducted on a total of 24 calf eyes and ten human eyes. Detailed results of these experiments (with the exception of the last pair of human eyes that were conducted more recently) can be found in Johnson (1987). Here we provide a summary.

Table II shows the repeatability of the measurement of microsphere concentration in six different solutions. In most cases the repeatability is reasonably good. The mean coefficient of variation was $21 \%$ for the $0.46-\mu \mathrm{m}$ microspheres and $6 \%$ for the $0 \cdot 176-\mu \mathrm{m}$ microspheres. These measurements also indicate that the solutions used in the different experiments had similar concentrations of microspheres.

For each eye, the percentage of microspheres (relative to the control solution) passing out of the outflow system could be determined as a function of time. No time-dependent change in the percentage of microspheres passing through the outflow system was observed for bovine or human eyes (Figs 1 and 2). (Note that for very short times the percentage passing through the system must be time-dependent, but samples from early times were rejected to prevent this dilution effect.) Given this result, the percentages measured at different times for a single eye were averaged to yield a single reading.

The percentages of 0.176 - and $0 \cdot 46-\mu \mathrm{m}$ microspheres passing through the individual eyes are presented in Table III. The values given are averaged over ' $m$ ' readings taken at different times. No significant difference was found depending on whether the infusion needle was in the posterior chamber or in the anterior chamber.

Table IV presents the pooled results for calf and human eyes, respectively, for which the control solution was measured using fluid obtained from the anterior chamber or from an exchange. A significant fraction of all sizes of microspheres was captured. Capture rates were found to be more than $90 \%$ in the calf eyes but only about $45 \%$ in the human eyes. No differences were apparent when carboxylate-labeled microspheres were used (experiments 17,26 and 29). [In experiment $29 \mathrm{a}, 3 \cdot 1 \pm 1 \cdot 5 \%(m=2)$ of the $0 \cdot 3-\mu \mathrm{m}$ microspheres passed through the outflow system, while in $29 \mathrm{~b}$ this valuc was $9.5 \%(m=1)$.

Smaller microspheres passed more readily through the calf outflow system than larger microspheres. The $0 \cdot 176-\mu \mathrm{m}$ microspheres passed through the calf outflow systcm with a frequency of $7 \cdot 7 \%$. compared to $2.5 \%$ for the $0.46-\mu \mathrm{m}$ microspheres; this difference was statistically significant with $P<0.02$, using the

TABLE II

Measurement repeatability of microsphere concentration (mean \pm s.E.)

\begin{tabular}{|c|c|c|c|c|c|}
\hline \multirow{2}{*}{$\begin{array}{l}\text { Experiment } \\
\text { None }\end{array}$} & Microsphere $(\mu \mathrm{m})$ & Solid fraction* & \multicolumn{3}{|c|}{ Microspheres/filter region } \\
\hline & $\begin{array}{l}0.46 \\
0.46\end{array}$ & $\begin{array}{l}3 \times 10^{-6} \\
4 \times 10^{-6}\end{array}$ & $\begin{array}{r}129 \pm 8 \\
95 \pm 7\end{array}$ & $\begin{array}{l}142 \pm 9 \\
127+9\end{array}$ & $126 \pm 8$ \\
\hline 5 & $\begin{array}{l}0.46 \\
0.176\end{array}$ & $\begin{array}{l}4 \times 10^{-6} \\
1 \times 10^{-6}\end{array}$ & $\begin{array}{c}95 \pm 7 \\
136+15\end{array}$ & $\begin{array}{l}127 \pm 9 \\
141 \pm 17\end{array}$ & - \\
\hline $7 a$ & $\begin{array}{l}0.176 \\
0.46\end{array}$ & $\begin{array}{c}1 \times 10^{-6} \\
2.4 \times 10^{-6}\end{array}$ & $\begin{array}{l}136 \pm 15 \\
105 \pm 18\end{array}$ & $\begin{array}{l}141 \pm 17 \\
177 \pm 21\end{array}$ & - \\
\hline $7 \mathrm{~b}$ & $0 \cdot 176$ & $1 \times 10^{-6}$ & $185 \pm 22$ & $211 \pm 17$ & - \\
\hline 8 & $0 \cdot 176$ & $1.6 \times 10^{-6}$ & $114 \pm 13$ & $89 \pm 7$ & $92 \pm 24$ \\
\hline 9 & $\begin{array}{l}0 \cdot 46 \\
0 \cdot 176\end{array}$ & $\begin{array}{l}3.2 \times 10^{-6} \\
1.6 \times 10^{-6}\end{array}$ & $\begin{array}{c}102 \pm 13 \\
89 \pm 7\end{array}$ & $\begin{array}{l}88 \pm 5 \\
87 \pm 4\end{array}$ & $48 \pm 13$ \\
\hline & $\begin{array}{l}0 \cdot 46 \\
1 \cdot 1\end{array}$ & $\begin{array}{r}3.2 \times 10^{-6} \\
2 \times 10^{-6}\end{array}$ & $\begin{array}{l}45 \pm 4 \\
40 \pm 3\end{array}$ & $\begin{array}{l}49 \pm 5 \\
40 \pm 4\end{array}$ & - \\
\hline
\end{tabular}

\footnotetext{
- Volume of microspheres/volume of solution.
} 


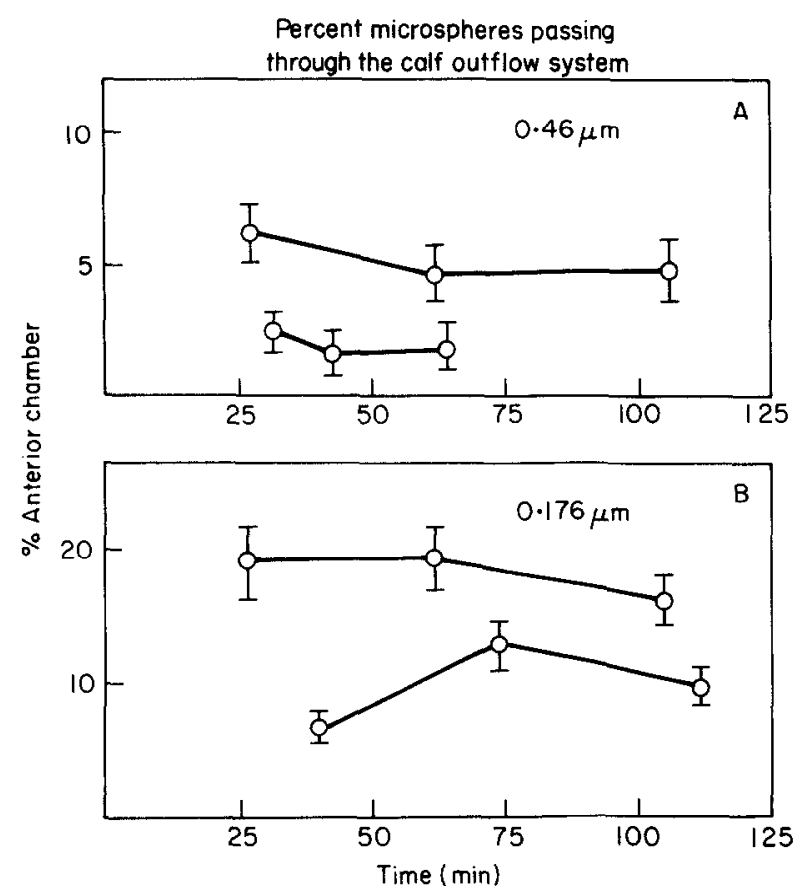

Fig. 1. Percentage of $0 \cdot 46-$ and $0 \cdot 176-\mu \mathrm{m}$ microspheres ( \pm S.E.) passing through the calf outflow system as a function of time. The upper curve in Figs $1(\mathrm{~A})$ and $(\mathrm{B})$ is from experiment 6a. The lower curve in Fig. 1(A) is from experiment 15a, while the lower curve in Fig. 1(B) is from experiment $6 \mathrm{~b}$.

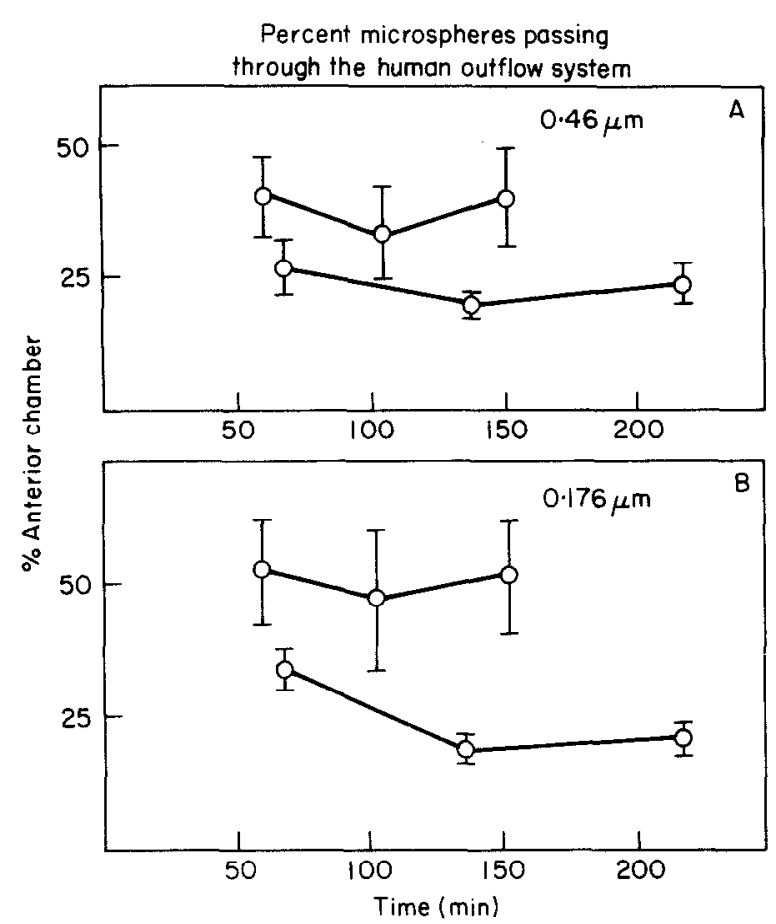

FIG. 2. Percentage of $0 \cdot 46$ - and $0 \cdot 176-\mu \mathrm{m}$ microspheres ( \pm S.E.) passing through the human outflow system as a function of time. The upper curve in each figure is from experiment 16. The lower curve in each is from experiment $7 \mathrm{a}$ and has been correct for anterior chamber dilution based on the results of experiment $7 \mathrm{~b}$.

two-sided Student's t-test. In eight eyes, in which $0 \cdot 176$ - and $0 \cdot 46-\mu \mathrm{m}$ microspheres were perfused simultaneously, the smaller microspheres were $2 \cdot 7$ times more likely to pass through the system than the larger microspheres, which was marginally different from unity with $P<0 \cdot 1$. For human eyes, no difference was found between the capture rates of these two microsphere sizes.

Noting that the calf aqueous outflow system captured significantly more microspheres of both sizes than did the human system $\mathbf{1 9 0 - 9 9 \%}$ in the calf vs. about $45 \%$ in the human), the effect of post-mortem time was explored. Experiments 1 and 30 were conducted on calf eyes perfused 1-2 days post-mortem. The percentages passing were similar to those of freshly perfused calf eyes and much lower than that characteristic of the human outflow system.

Experiment 29 was conducted to verify the result of other investigators that large microspheres can successfully pass through the outflow system. In $29 \mathrm{a}$, $2 \cdot 6 \pm 0 \cdot 6 \%(m=2)$ of the $1 \cdot 1-\mu \mathrm{m}$ microspheres passed through the calf outflow system while in $29 \mathrm{~b}$, this value was $1.2 \%(m=1)$.

The data for which only a Baseline control solution was taken (experiments 1-5 and 7a) were not used in the pooled data analysis. Nonetheless, these data were consistent with the other results. This is not surprising as most of the microsphere solution enters the anterior chamber at the start of an experiment and thus the concentration of microspheres in the anterior chamber remains nearly constant (within a factor of two) during the course of the experiment.

For the single pair of glaucomatous eyes investigated (experiment 8 ), 6.8 and $3.6 \%$ of the 0.176 - and $0 \cdot 46-\mu \mathrm{m}$ microspheres. respectively, passed through the outflow system. This experiment was unusually long ( $\sim 15 \mathrm{hr}$ ) due to the low flow rate that resulted from the low outflow facility of this pair of eyes [0.02-0.03 ( $/ \mathrm{l} \mathrm{min}) /(\mathrm{mmHg})]$.

\section{Morphological Resulls}

A study was performed to determine the dimensions of the calf outflow system to aid in the interpretation of the filtration data. The calf outflow system (Fig. 3) has an extensive 'uveal meshwork' with relatively large open spaces known as the spaces of Fontana; only the anterior portion appears to carry the aqueous outflow (K. Erickson-Lamy, pers. comm.). The aqueous humor then passes through the calf's equivalent of the trabecular meshwork, a reticular meshwork (Rohen, 1986) which lacks the organized network of trabeculae found in the human eye (see Fig. 4 and Grierson et al., 1985). The distance from the uveal region to the angular aqueous plexus (the bovine system analogous to Schlemm's canal ; Tripathi, 1974) is less in the calf than in the human, averaging about $50 \mu \mathrm{m}$ when fixed at physiological pressure.

An area analogous to the JCT is difficult to distinguish from the reticular meshwork: the openings are about the same size as those in the remainder of the reticular meshwork $(5-10 \mu \mathrm{m})$. This region was 
TABLE III

Percentage of $0 \cdot 176-\mu \mathrm{m}$ and $0 \cdot 46-\mu \mathrm{m}$ microspheres passing through the aqueous outflow system

\begin{tabular}{|c|c|c|c|}
\hline \multirow[b]{2}{*}{ Experiment } & \multicolumn{3}{|c|}{ Percent passing $( \pm$ S.E. $)$} \\
\hline & $0 \cdot 176$ & $0 \cdot 46$ & $0 \cdot 176 / 0 \cdot 46$ \\
\hline \multicolumn{4}{|l|}{ Calf eyes } \\
\hline $1 \mathrm{a}$ & - & $5 \cdot 8 \pm 1 \cdot 9(m=3)$ & - \\
\hline $1 \mathrm{~b}$ & - & $14 \cdot 0 \pm 4 \cdot 4(m=3)$ & - \\
\hline $3 a$ & - & $4 \cdot 7 \pm 0 \cdot 4(m=4)$ & - \\
\hline $3 b$ & - & $1.9 \pm 0.2(m=3)$ & - \\
\hline $4 \mathbf{a}$ & $14 \cdot 3 \pm 4 \cdot 0(m=3)$ & - & - \\
\hline $4 b$ & $5 \cdot 5 \pm 0 \cdot 2(m=2)$ & - & - \\
\hline $6 a$ & $18 \cdot 1 \pm 1 \cdot 0(m=3)$ & $5 \cdot 1 \pm 0.5(m=3)$ & $3.6 \pm 0.3(m=3)$ \\
\hline $6 \mathrm{~b}$ & $9 \cdot 6 \pm 1 \cdot 8(m=3)$ & - & - \\
\hline $10 a$ & $11 \cdot 2 \pm 2 \cdot 6(m=2)$ & - & - \\
\hline $10 \mathrm{~b}$ & $6 \cdot \overline{3} \quad(m=1)$ & - & - \\
\hline $12 \mathrm{a}$ & $2 \cdot 4 \pm 0.5(m=2)$ & $3 \cdot 6 \pm 1 \cdot 2(m=2)$ & $0 \cdot 7 \pm 0 \cdot 1(m=2)$ \\
\hline $12 b$ & $3 \cdot 3 \pm 1 \cdot 6(m=3)$ & $2.7 \pm 0.5(m=3)$ & $1.5 \pm 0.8(m=3)$ \\
\hline $13 a$ & $3.9 \pm 2.5(m=2)$ & $2 \cdot 3 \pm 0 \cdot 3(m=2)$ & $1.6 \pm 0.9(m=2)$ \\
\hline $13 \mathrm{~b}$ & $0.4 \pm 0.4(m=2)$ & $0 \cdot 7 \pm 0 \cdot 1(m=2)$ & $0.5 \pm 0.5(m=2)$ \\
\hline $14 \mathrm{a}$ & $11.4 \pm 3.9(m=3)$ & $1.6 \pm 0.6(m=3)$ & $7 \cdot 4 \pm 0.9(m=3)$ \\
\hline $15 \mathrm{a}$ & $7 \cdot 8 \pm 2 \cdot 6(m=3)$ & $1.9 \pm 0 \cdot 2(m=3)$ & $3 \cdot 8 \pm 0 \cdot 8(m=3)$ \\
\hline $15 b$ & $10 \cdot 4 \pm 5 \cdot 2(m=3)$ & $3 \cdot 6 \pm 1 \cdot 7(m=3)$ & $2 \cdot 6 \pm 0 \cdot 6(m=3)$ \\
\hline $17 \mathrm{a}$ & - & $0 \cdot 8 \pm 0 \cdot 2(m=2)$ & - \\
\hline $26 a$ & $\cdots$ & $2 \cdot 9(m=1)$ & - \\
\hline $26 \mathrm{~b}$ & - & $0 \cdot 9(m=1)$ & - \\
\hline $26 c$ & - & $1 \cdot 7(m=1)$ & - \\
\hline $26 \mathrm{~d}$ & - & $3 \cdot 1(m=1)$ & - \\
\hline $30 a$ & $6 \cdot 0(m=1)$ & $2 \cdot 9(m=1)$ & $2 \cdot 1(m=1)$ \\
\hline $30 b$ & $8 \cdot 9(m=1)$ & $4 \cdot 0(m=1)$ & $2 \cdot 2(m=1)$ \\
\hline \multicolumn{4}{|l|}{ Human eyes } \\
\hline $2 \mathrm{a}$ & - & $15 \cdot 2(m=1)$ & \\
\hline $2 b$ & - & $25 \cdot 0 \pm 11 \cdot 1(m=2)$ & - \\
\hline $5 a$ & - & $35.5 \pm 3.5(m=2)$ & - \\
\hline $7 a$ & $14 \cdot 0 \pm 2 \cdot 7(m=3)$ & $13 \cdot 2 \pm 1 \cdot 2(m=3)$ & $1 \cdot 1 \pm 0 \cdot 1(m=3)$ \\
\hline $7 \mathrm{~b}$ & $41(m=1)$ & - & - \\
\hline $8 \mathrm{a}$ & $6 \cdot 8 \pm 0 \cdot 7(m=3)$ & $3 \cdot 6 \pm 0 \cdot 8(m=3)$ & $2 \cdot 2 \pm 0 \cdot 6(m=3)$ \\
\hline $16 \mathbf{a}$ & $51 \cdot 0 \pm 1 \cdot 5(m=3)$ & $37 \cdot 3 \pm 2 \cdot 2(m=3)$ & $1 \cdot 4 \pm 0 \cdot 1(m=3)$ \\
\hline $32 \mathrm{a}$ & $69 \cdot 0 \pm 16 \cdot 0(m=3)$ & $55 \cdot 7 \pm 12 \cdot 8(m=3)$ & $1 \cdot 3 \pm 0 \cdot 1(m=3)$ \\
\hline $34 a$ & $39.5 \pm 1.5(m=2)$ & $42 \cdot 0 \pm 2 \cdot 0(m=2)$ & $0.9 \pm 0.0(m=2)$ \\
\hline $34 b$ & $76.5 \pm 3.5(m=2)$ & $76.5 \pm 3.5(m=2)$ & $1 \cdot 0 \pm 0 \cdot 0(m=2)$ \\
\hline
\end{tabular}

$m$ is the number of measurements.

\section{TABLE IV}

Summary of percent passage of $0 \cdot 176-\mu \mathrm{m}$ and $0 \cdot 46-\mu \mathrm{m}$ microspheres

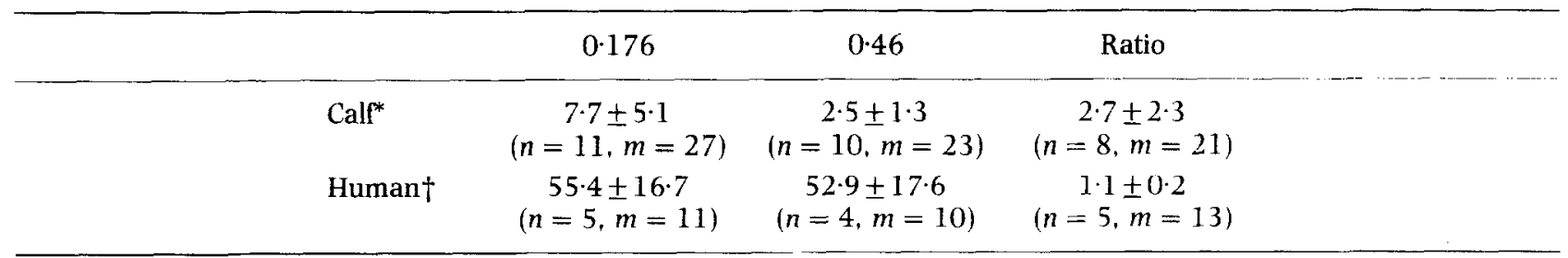

$n$ : number of eyes, $m$ : number of measurements.

* Data from eyes $6,10,12,13,14,15,26 \mathrm{a}, \mathrm{b}$.

+ Data from eyes $7 \mathrm{a}$ (ratio), $7 \mathrm{~b}, 16,32,34$

The uncertainties reported in this table are standard deviations (rather than standard errors) as discussed in the text.

determined to be $15 \pm 5 \mu \mathrm{m}$ in length measuring from the first beam-like structure to the inner wall endothelium.

From the meshwork, the aqueous passes across a continuous endothelial cell layer into the vessels of the angular aqueous plexus. Focal breaks were occasionally observed in the inner wall of the angular aqueous plexus [Fig. 4(A)]; one of the authors (AWdK) 


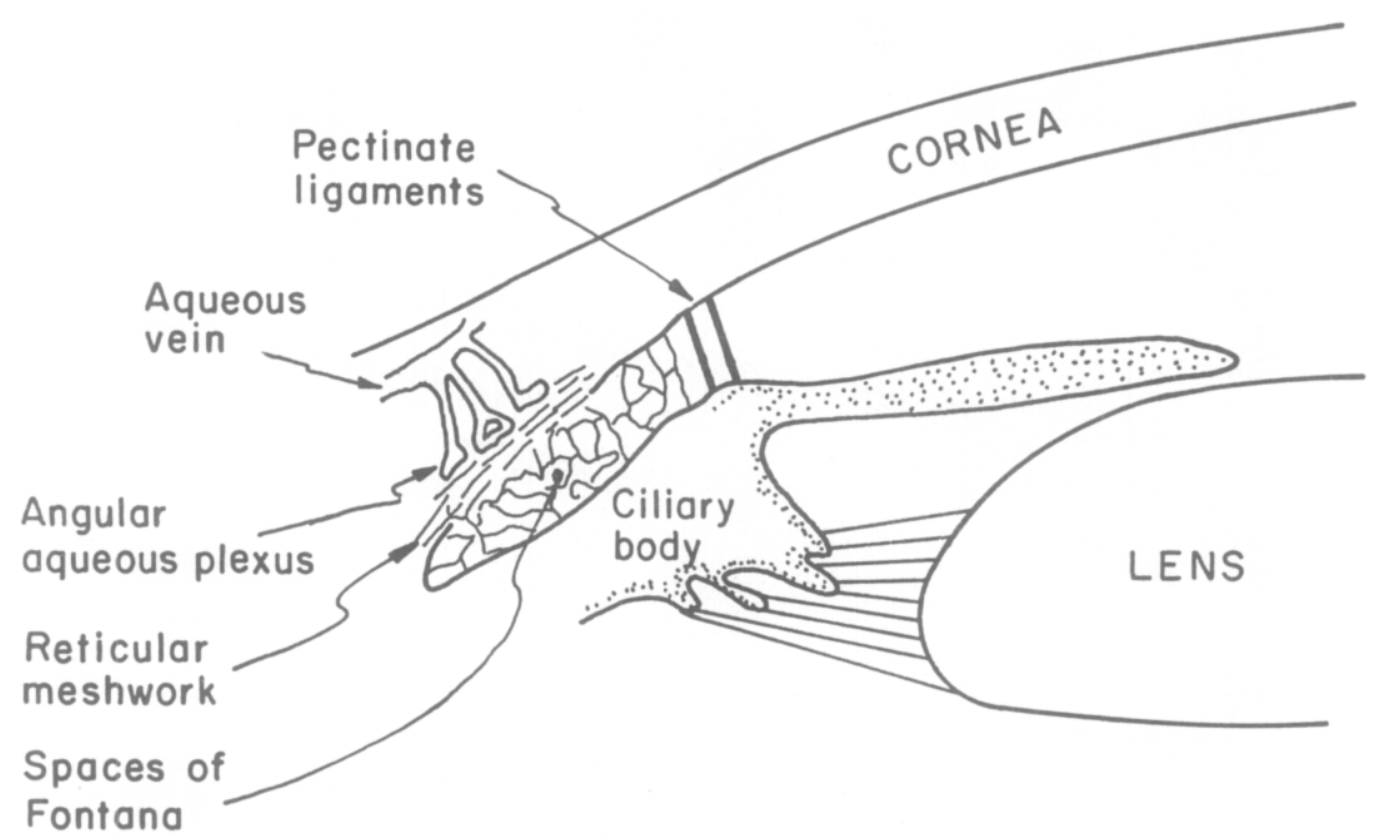

FIG. 3. Schematic of calf outflow system.

has observed that this is normal in bovine eyes, fixed at pressure, that have not undergone experimental manipulation.

Two to three vessels in the sclera comprise a system anatomically analogous to Schlemm's canal, the combined width of which is $450 \pm 150 \mu \mathrm{m}$. These vessels travel circumferentially around the eyewhere one stops, another usually continues. The circumference of the calf iris is approximately $5.5 \mathrm{~cm}$ and thus the cross-sectional area facing flow (at the level of the angular aqueous plexus) is approximately $0 \cdot 25 \mathrm{~cm}^{2}$. Note that, with the exception of the radial dimension of the trabecular meshwork, all linear dimensions of the calf outflow system are approximately 1.6 times as large as their human equivalent.

A total of four calf eyes and four human eyes were examined morphologically to determine the distribution of microspheres captured by the outflow system. In the calf meshwork, microspheres $(0.5 \mu \mathrm{m}$ carboxylate-labeled) were captured throughout the system [Fig. 4(A, B)] although a greater number of microspheres were found in the inner meshwork than in the deeper aspects of this tissue. In the human outflow system, microspheres $(0 \cdot 46-\mu \mathrm{m}$ carboxylatelabeled and $0 \cdot 176-\mu \mathrm{m}$ microspheres) were captured throughout the trabecular meshwork and JCT [Fig. $5(A-C)]$. A slightly greater number appeared to be caught in the latter region, although no large accumulation of microspheres was observed.

\section{Theoretical Analysis}

As mentioned above, there are two fundamental mechanisms by which the microspheres might be captured as they attempt to pass through the aqueous outflow system: (i) steric hindrance when the microsphere is too large to pass through a pore it encounters, and (ii) capture by an interaction between the microsphere and the filtering surface ("sticky wall' capture). In this section, we use simple analytical models to characterize these two capture modes; in the discussion section, we then compare the predictions of the models to the microsphere capture data.

\section{Capture by Steric Hindrance}

We model microsphere capture as occurring in an idealized filter having parallcl, circular pores of various diameters. A function $f(r)$ is introduced that charactcrizes the pore size distribution in the filter such that the fraction of pores with a radius between $r_{1}$ and $r_{2}$ is:

$$
\int_{r_{1}}^{r_{2}} f(r) d r .
$$

As a microsphere attempts to pass through the filter. the probability of it being carried toward a pore of a given radius depends not on the fraction of pores of that size, but rather on the amount of flow passing through that pore size. This will make the microsphere more likely to enter the larger pores as they carry more of the flow.

In order to weight the pore distribution to properly account for this effect, we define a probability density function (PDF) $f^{*}(r)$ that assumes the flow through each pore to be weighted by the fourth power of the radius (Poiseuille's law). Thus, the probability distribution $f(r)$ is multiplied by $r^{4}$ and then normalized such that the integral of $f^{*}(r)$ over all pore sizes is unity :

$$
f^{*}(r)=\frac{r^{4} f(r)}{\int_{0}^{\infty} r^{4} f(r) \mathrm{d} r},
$$




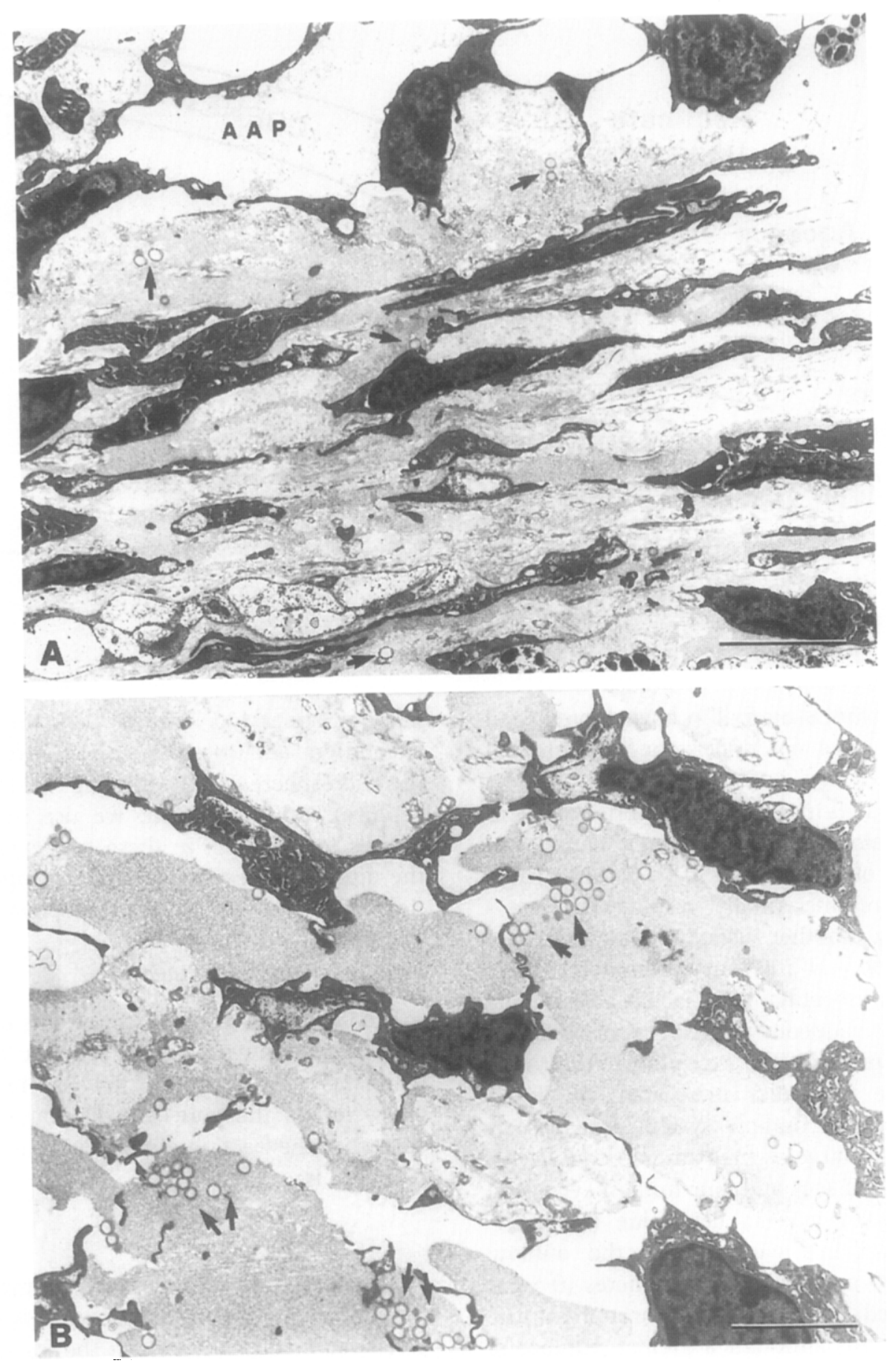

FIG. 4. Transmission electron micrograph of calf meshwork perfused with $0.5-\mu \mathrm{m}$ microspheres and gelatin. (A) Angular aqueous plexus (AAP) and subendothelial meshwork. (B) Mid meshwork region showing capture of a large number of microspheres. Line bars: $5 \mu \mathrm{m}$.

$\mathrm{f}^{*}(r)$ is the probability that a microsphere will try to enter a pore of size $r$. We now further assume that a microsphere of radius $R$ will successfully pass through the pore if $R<r$. Then, the probability of a particle passing through the filter becomes:

$$
\mathrm{P}(R)=\frac{\int_{R}^{\infty} r^{4} \mathrm{f}(r) \mathrm{d} r}{\int_{0}^{\infty} r^{4} \mathrm{f}(r) \mathrm{d} r} .
$$

The probability function $\mathrm{P}(R)$ can be compared to the results for microsphere passage through the aqueous outflow system. Figure 6 presents the results of Huggert et al. (1955) showing the relative percentage of different sizes of particles passing through the aqueous outtlow system. 'These results indicate that $\mathrm{P}(R) \sim R^{-1}$. Comparing this result with eqn (2). we can find that $\mathrm{f}(r)$ must go as $R^{-6}$ : there must be $10^{6}$ more pores $0.15 \mu \mathrm{m}$ in diameter than $1.5 \mu \mathrm{m}$ in diameter. This provides analytical support for the 

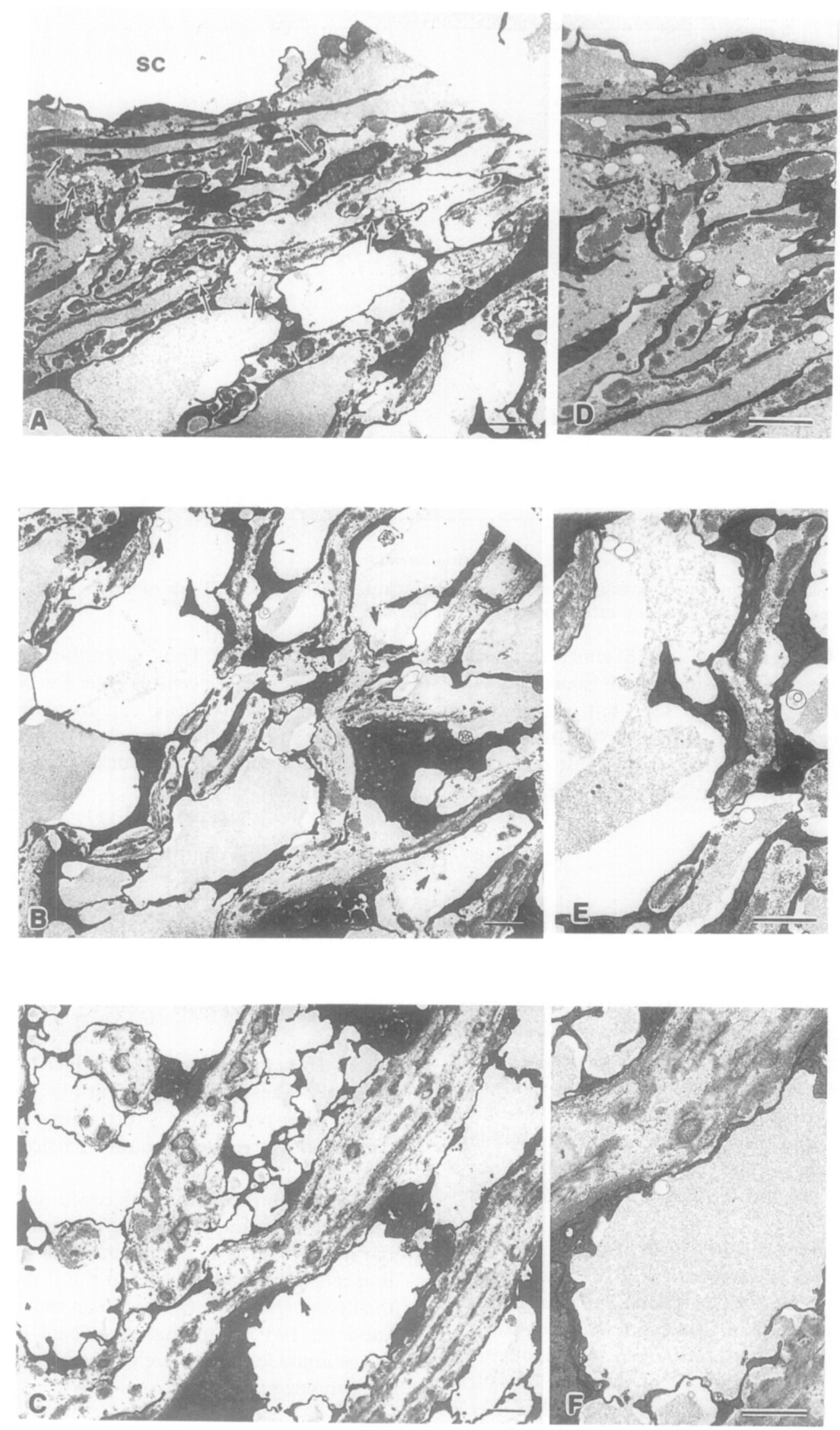

Fig. 5. Transmission electron micrograph of human meshwork perfused with $0 \cdot 176$ - and $0 \cdot 5-\mu \mathrm{m}$ microspheres and gelatin. Microspheres are captured throughout the juxtacanalicular tissue (A), corneoscleral meshwork (B) and uveal meshwork (C). It appears that a slightly greater number of microspheres are caught in the juxtacanalicular region. (D), (E) and (F) are high magnification inserts of selected areas where microspheres are captured in (A), (B), and (C), respectively. SC, Schlemm's canal. Line bars: $1 \mu \mathrm{m}$. 


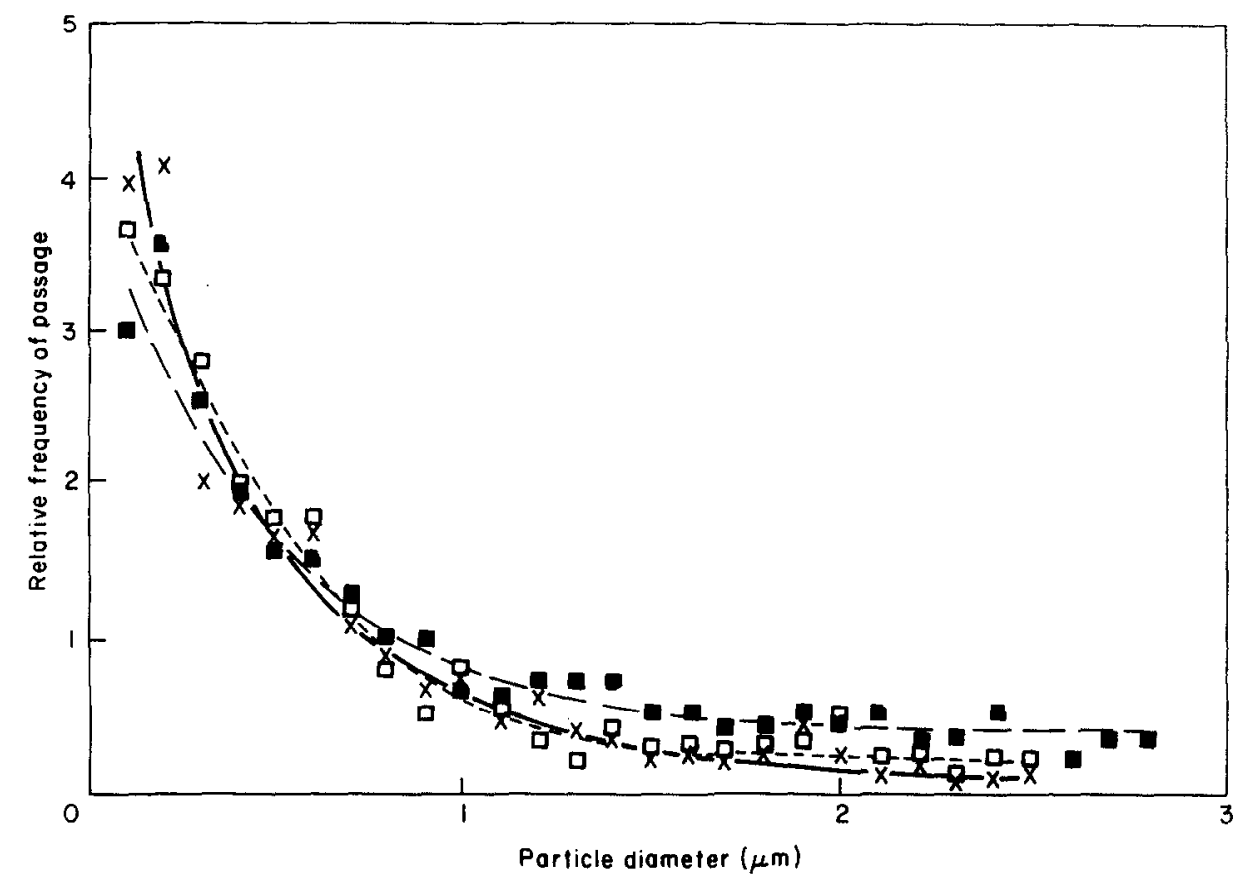

FiG. 6. The relative frequency of passage of particles through cattle $(\boldsymbol{D})$, human, $(\square)$, or rabbit $(\times)$ eyes as a function of particle diameter. Units of ordinate are arbitrary (Huggert et al., 1955)

conclusion of Huggert et al. (1955) that: 'the results given here cannot be explained by an isoporosity with all pores of the maximal size, but rather by a heteroporosity in which, however, the distribution curve seems to be such that the greatest part of the pores lies within the region below the avcrage values found'.

\section{Capture by 'Sticky Walls'}

As mentioned, Barany (1959) sought an alternative interpretation to Fig. 6 : he proposed that the particles are captured by 'sticky walls'. The process by which a particle is captured involves electrostatic interactions, induced electrostatic interactions and London dispcrsive forces (Spielman, 1977). Specific biologic binding processes (e.g. fibronectin) may also be important. Here we model a 'sticky wall' by assuming that all microspheres that come in contact with the wall adhere to it, and thus the concentration of microspheres at the wall is assumed to be zero (Spielman, 1977).

We again represent the filtration region as a set of parallel pores. A microsphere will be captured if its path intercepts the pore wall upon entry, or if during transit through the pore, Brownian motion causes it to impact the wall. Barany (1959) followed this approach when analyzing the capture of particles by 'sticky walls' but the expression he used to characterize particulate capture due to Brownian motion was incorrect (see below).

The velocity profile at the entrance to a round pore is nearly parabolic (Poiseuille-like) for viscous flow conditions (Dagan. Weinbaum and Pfeffer, 1982a). and the velocity of a microsphere entering the pore is similar to that of the local fluid (Dagan. Weinbaum and Pfeffer, 1982b). Thus, given the condition that all microspheres whose centres enter the pore at a radius greater than $r-R$ will be captured (the microsphere will hit the pore wall upon entry), the probability $\left(\mathrm{P}_{1}\right)$ of a particle entering the sticky pore without being captured is :

$$
P_{1}=2(1-R / r)^{2}-(1-R / r)^{4}
$$

To describe the capture of the microspheres as they pass through the pores, Johnson (1987) used the convection-diffusion equation to characterize microsphere transport. He found the probability of successfully passing through the pore $\left(\mathrm{P}_{2}\right)$ to be:

$$
P_{2} \simeq 0.82 \exp \left[\frac{-3.7 D \epsilon V}{Q(r-R)^{2}}\right]
$$

where $D$ is the microsphere diffusivity (determined from the Stokes-Einstein relationship; Einstein, 1908), $\epsilon$ is the filter porosity, $V$ is the volume of the filtration region and $Q$ is the flow rate passing through the system.

The total probability of successful passage through the filter is found as $\mathrm{P}_{1}{ }^{*} \mathrm{P}_{2}$. The expression used by Barany (1959) was similar but had the quantity in brackets [in eqn (4)] raised to the $\frac{1}{2}$ power instead of the first power (this was due to an error in a paper by Landahl, 1950). This has significant implications as the estimate for a pore size of $1 \mu \mathrm{m}$ given by Barany is thus somewhat low.

\section{Discussion}

The work of our group and others has indicated that the characteristic flow dimensions responsible for generating aqueous outflow resistance were not determinable from morphological studies of the aqueous outflow system (McEwen, 1958; Bill and 
Svedbergh, 1972; Johnson and Kamm, 1983; Ethier et al., 1986). Seeking alternative methods to determine the dimensions of the flow limiting passages, we investigated the use of filtration studies.

Huggert (1955) had previously shown that small particles $(\sim 0 \cdot 1 \mu \mathrm{m})$ pass through the outflow system more readily than do large particles $(\sim 2.5 \mu \mathrm{m})$ (see Fig. 6). However, in his studies, the particles were suspended in distilled water thus placing a tremendous osmotic stress on the cells of the outflow system. Nonetheless, using $0 \cdot 176$ - and $0 \cdot 46-\mu \mathrm{m}$ microspheres in bovine eyes, we confirmed this observation and showed that there was significant capture of both sizes of microspheres. This was an important observation as Huggert had presented only relative frequency of passage, rather than the absolute value.

One goal of this study was to see if microspheres that were smaller thran the smallest openings seen morphologically in the outflow system pass freely out of the eye. The $0 \cdot 176-\mu \mathrm{m}$ microspheres are smaller than most of the openings seen in the trabecular meshwork or JCT, but $92 \%$ of them were captured by the calf outflow system, while $45 \%$ of them were captured by the human system.

Thus we can conclude that the capture of microspheres is not due to steric hindrance by the morphologically observed openings in the outflow system. This result is clearly demonstrated in Figs 4 and 5, showing microspheres captured in openings much larger than the microsphere diameter.

In contrast to the study of Inomata et al. (1972), the infusion of gelatin followed the perfusion of microspheres through the outflow system. This procedure should remove microspheres in transit, but it would not be expected to dislodge the previously caught microspheres as they had already been subject to several hours of perfusion. Microspheres were captured throughout the aqueous outflow system; in the calf, there was a tendency for more to be captured in the proximal aspects of the reticular meshwork, while in the human there was some accumulation in the JCT. However, in both species, a large fraction of the microspheres were captured in regions that were presumably unrelated to the generation of aqueous outflow resistance. This observation could also explain how the calf eye, with a somewhat higher outflow facility per unit cross-sectional area than the human eye, could nonetheless have a much higher filtration efficiency. These results suggested that the site of filtration may be distinct from the site of flow resistance.

To the extent that 'sticky walls' capture, rather than steric hindrance, determines the filtration characteristics of the aqueous outflow system, eqns (3) and (4) should provide some indication of system behavior. For the calf, we use a flow rate of $20 \mu \mathrm{min}^{-1}$ and the morphological parameters given above. Assuming that capture occurs throughout the reticular meshwork, the data for capture of the $0 \cdot 176-\mu \mathrm{m}$ is consistent with a pore diameter of between 5 and $10 \mu \mathrm{m}$; however, the model predicts too low a capture rate for the $0.46-\mu \mathrm{m}$ microspheres (40-60\% when, in fact, 97.5\% were captured). Use of a smaller pore size (such as Barany's prediction of a $1-\mu \mathrm{m}$ pore size) leads to a prediction of nearly complete capture of both sizes of microspheres. There is no single pore size that can be used with eqns (3) and (4) that is consistent with the experimental data.

The experimental result that more $0.46-\mu \mathrm{m}$ microspheres are captured than $0 \cdot 176-\mu \mathrm{m}$ microspheres cannot be due entirely to sticky wall capture. The higher predicted capture rate [eqn (4)] for the $0 \cdot 176-\mu \mathrm{m}$ microspheres is due to their enhanced Brownian motion compared with that of the $0.46-\mu \mathrm{m}$ microspheres. Only for very short pores (a few microns long) would the $0 \cdot 176-\mu \mathrm{m}$ microspheres have a lower capture rate than the $0 \cdot 46-\mu \mathrm{m}$ microspheres. However, the morphological evidence shows microsphere capture throughout the calf meshwork, not in a short region.

While steric hindrance by the morphologically observable openings can be ruled out, there must be another way by which steric hindrance can play an important role. It is widely believed that an extracellular matrix gel fills some portion of the open spaces of the trabecular meshwork and/or the JCT (Ethier et al., 1986). This gel would have a large array of pore sizcs with the vast majority being much smaller than $0.176 \mu \mathrm{m}$, and thus this gel may capture microspheres as they pass through these regions. In this way, the predictions given at the end of the section on capture by steric hindrance (that of many more small porcs than large pores) could still be met. If the gel filling the extracellular space is not uniform, then there may be more gel-free pathways accessible to the $0.176-\mu \mathrm{m}$ microspheres than to the $0.46-\mu \mathrm{m}$ microspheres; such a system would be consistent with the data and thus microsphere capture in the calf outflow system would be due to a combination of steric hindrance and 'sticky walls'.

In the human eye the morphology is quite different. The trabecular meshwork has large openings that decrease in size from the uveal meshwork to the JCT (Tripathi, 1974) in contrast to the calf eye that has a relatively constant opening size. To model 'sticky wall' capture in the human outflow system, we treat the trabecular meshwork and the JCT separately. Using a flow rate of $5 \mu \mathrm{l} \mathrm{min}^{-1}$ (perfusion of enucleated eyes at $15 \mathrm{mmHg}$ ), a pore size of $10-30 \mu \mathrm{m}$ in the trabecular meshwork and $6 \mu \mathrm{m}$ in the JCT. the model predictions are consistent with the data for both 0.176 - and $0.46-\mu \mathrm{m}$ microspheres. The model predicts some accumulation of microspheres in the JCT although capture throughout the trabecular meshwork is predicted. These results are consistent with the morphological findings (see Fig. 5). Thus in the human eye, the theoretical prediction based on 'sticky wall' capture alone is consistent with the experimental results: however, steric capture due to gel-filled spaces cannot be ruled out. 
While a predicted pore size of $6 \mu \mathrm{m}$ in the human JCT may seem large, it must be recalled that most pore size studies of the ICT have been done on eyes that were fixed by immersion (e.g. Lütjen-Drecoll, Futa and Rohen, 1981). In eyes fixed at pressure (e.g. Johnstone and Grant, 1973), the spaces in the JCT are much larger. Lindenmayer et al. (1983) fixed primate eyes at $15 \mathrm{mmHg}$ (similar to the experimental conditions of the present study) and found a porosity of 0.6 , almost three times the values in eyes fixed by immersion (Ethier et al., 1986).

Results from the present study may be used to reconcile an apparent discrepancy between the data of Karg et al. (1959) showing no capture in human eyes for microspheres smaller than $1.5 \mu \mathrm{m}$ and those of Huggert (1955) and Huggert et al. (1955) showing significant capture for all particle sizes studied. We found that roughly half of the microspheres entering the human outflow system were captured. Karg et al. (1959) used the following measurement scheme to characterize the microsphere concentration in solution : $(+++)$ represented the same concentration as the original perfusion fluid, $(++)$ slightly less, and $(+)$ markedly less. It may be that this grading scheme would not differentiate between solutions with a twofold difference in concentration [keeping in mind that $(+)$ might have to be reserved for solutions with less than $1 \%$ of control]. Perhaps in this fashion can the differences with Karg et al. (1959) be reconciled.

In the single glaucomatous eye $(n=8)$ studied there was a very high capture rate of microspheres compared to that of normal human eyes. It could not be determined whether this result was due to the extremely low flow rate [see eqn (4)] or an increased gel concentration and concomitant decreased interfiber spacing. Thus, both models are consistent with the results of this single glaucomatous eye.

In this study, we have found that microspheres are captured by 'sticky' wall capture and perhaps also by the extracellular matrix filling the open spaces. Thus the site of filtration may be distinct from the site of flow resistance. A disappointing corollary is that filtration studies will provide little information concerning the dimensions of the flow limiting passages in the aqueous outflow system. New methods will have to be developed to determine this important parameter.

\section{Acknowledgments}

We gratefully acknowledge many helpful discussions with Dr W. Morton Grant. This study was supported by a grant from the National Eye Institute R01 EY05503 and by the National Glaucoma Research Program, part of the American Health Assistance Foundation.

\section{References}

Barany, E. (1959). Pore size and passage of particulate matter through the trabecular meshwork. Doc. Ophthalmol. 13, 41-55.
Bill, A. and Svedbergh, B. (1972). Scanning electron microscopic studies of the trabecular meshwork and the canal of Schlemm - an attempt to localize the main resistance to outflow of aqueous humor in man. Acte Ophthalmol. 50, 295-319.

Dagan, Z. Weinbaum, S, and Pfeffer, K. (1982a). An infinite-series solution for the creeping motion through an orifice of finite length. J. Fluid Mech. 115. 505-23.

Dagan, Z., Weinbaum, S. and Pfeffer, R. (1982b). General theory for the creeping motion of a finite sphere along the axis of a circular orifice, J. Fluid Mech. 117. 143-70.

Einstein, A. (1908). The elementary theory of brownian motion. Z. Elektrochemie 14, 235-9.

Ethier, C. R., Kamm, R. D., Palaszewski, B. A., Johnson. M. and Richardson, T. M. (1986). Calculations of flow resistance in the juxtacanalicular meshwork. Invest. Ophthalmol. Vis. Sci. 27, 1741-50.

Grierson, I., Robins, E.. Unger, W., Millar, L., and Ahmed, A. (1985). The cells of the bovine outflow system in tissue culture, Exp. Eye Res. 40, 35-46.

Huggert, A. (1955). Pore size in the filtration angle of the eye, Acta Ophthalmol. 33, 271-84.

Huggert, A. (1957). An experiment in determining the pore size distribution curve to the filtration angle of the eye. Acta Ophthalmol. 35, 12-19, 104-112.

Huggert, A., Holmberg, A. and Esklund, A. (1955). Further studies concerning the filtration angle of the eye. Acta Ophthalmol. 33, 429-36.

Inomata, H., Bill, A. and Smelser, G. K. (1972). Aqueous humor pathways through the trabecular meshwork and into Schlemm's canal in the cynomolgus monkey (Macaca Irus). Am. J. Ophthalmol. 73, 760-89.

Johnson, M. (1987). Transport through the Aqueous Outflow System of the Eye. PhD thesis, Department of Mechanical Engineering, MIT.

Johnson, M. and Kamm, R. D. (1983). The role of Schlemm's canal in aqueous outflow from the human eye. Invest. Opthalmol. Vis. Sci. 24, 320-5.

Johnstone, M. A. and Grant, W. M (1973). Pressure dependent changes in the structures of the aqueous outflow system of human and monkey eyes. Am. J. Ophthalmol. 75, 365-83.

Karg, S. J.. Garron, L. K., Feeney, L. and McEwen, W. K. (1959). Perfusion of human eyes with latex microspheres, Arch. Ophthalmol. 61, 68-71.

Landahl, H. D. (1950). On the removal of air-borne droplets by the human respiratory tract: 1 . The Lung. Bull. Math. Biophys. 12. 43--56.

Lindenmayer, J. M., Kahn, M. G., Hertzmark, E. and Epstein, D. L. (1983). Morphology and function of the aqueous outflow system in monkey eyes perfused with sulfhydryl reagents. Invest. Ophthalmol. Vis. Sci. 24, 710.

Lütjen-Drecoll, E., Futa, R. and Rohen, J. W. (1981). Ultrahistochemical studies on tangential sections of trabecular meshwork in normal and glaucomatous eyes. Invest. Ophthalmol. Vis. Sci. 21, 563-73.

McEwen, W. K. (1958). Application of Poiseulle's law to aqueous outflow, A.M.A. Arch. Ophthaimol. 60 , 290.

Moses, R. A. (1977). The effect of intraocular pressure on resistance to outflow. Surv. Ophthalmol. 22, 88-100.

Rohen, J. W. (1986). Anatomy of aqueous outflow channels. In Glaucoma (Ed. Cairns. J. E.). Vol. 1. Pp. 277-96. Grune and Stratton: London.

Spielman, L. A. (1977). Particle capture from low-speed laminar flows, Annu. Rev. Fluid Mech. 9, 297-319.

Tripathi, R. C. (1974). Comparative aspects of aqueous outflow. In The Eye. Vol. 5: Comparative Physiology (Ed. H. Davson). Chapter 3. Academic Press: London: New York. 